\title{
Comparison of rates of infection of two methods of emergency ventricular drainage
}

\author{
D K Kim, D Uttley, B A Bell, H T Marsh, A J Moore
}

\begin{abstract}
The rates of infection of two methods of external ventricular drainage in use at Atkinson Morley's Hospital-namely, (a) percutaneous drainage with Rickham reservoirs and (b) tunnelled ventriculostomies-were compared in this retrospective review. Percutaneous drainage of CSF with Rickham reservoirs was associated with a $\mathbf{2 7 \%}$ rate of infection as identified by positive microbiological cultures; tunnelled ventriculostomy catheters had a $10 \%$ infection rate. The difference in the infection rate between the two methods was statistically significant $(P<0.015)$. Other variables examined, including the age and sex of the patients and the reasons for ventricular drainage, were not associated with an increased rate of infection. Most infections from either method were caused by a coagulase negative staphylococcus. The average duration of ventricular drainage before identification of positive cultures was 5.7 days for Rickham reservoirs and 6.0 days for ventriculostomies.
\end{abstract}

(F Neurol Neurosurg Psychiatry 1995;58:444-446)

Keywords: infection; Rickham reservoir; tunnelled ventriculostomy

Intraventricular catheters were among the first techniques used to measure intracranial pressure. ${ }^{12}$ Albeit invasive, its accuracy is such that this technique serves as the standard to which other methods of measurement of intracranial pressure are compared. Ventricular drainage has also proved to be useful for temporary control of raised intracranial pressure, caused by trauma or hydrocephalus, ${ }^{1-5}$ especially in the management of the acute phase associated with subarachnoid haemorrhage, closed head injury, intracerebral and intraventricular haemorrhage, and neoplasms obstructing the circulation of CSF.

Infection is an important complication of ventricular drainage and directly related to its duration. Previously reported rates of infection of external ventricular catheters have ranged from 5\% to $30 \% .^{1467-9}$ Infected catheters may lead to meningitis, ventriculitis, subdural empyema, intracerebral abscess, and systemic sepsis. Aside from the associated mortality and morbidity, the practical consequences of infection are cost and morbidity associated with antibiotic treatment, prolonged stay in hospital, and additional, often invasive, procedures necessary for the management of infected catheters and their complications.

Two different methods of ventricular drainage have been employed at Atkinson Morley's Hospital: (a) percutaneous drainage with an in situ reservoir, popularised by Rickham and others, ${ }^{57}$ and (b) tunnelled ventriculostomy. ${ }^{610}$ In this retrospective analysis the infection rates of the methods are compared.

\section{Patients and methods}

The records of consecutive patients undergoing placement of intraventricular catheters for external CSF drainage at Atkinson Morley's Hospital over a three year period ending in March 1994 were reviewed. A total of 66 procedures (involving 61 patients) for Rickham reservoir placement and 70 procedures (involving 61 patients) for ventriculostomy was identified. Only those procedures leading to actual CSF drainage, shown by laboratory and microbiological analysis recorded in the notes, were retained for review. Ten of the Rickham reservoirs and five ventriculostomies were excluded from analysis due to pre-existing infection. One Rickham reservoir and one ventriculostomy catheter were excluded from the study because of inadequate records. The records of five patients could not be located.

Catheter placement for percutaneous CSF drainage was performed in standard fashion. ${ }^{57}$ The indwelling Rickham reservoir (Codman; Randolph, MS, USA) was placed into the frontal horn of the lateral ventricle $1 \mathrm{~cm}$ anterior to the coronal suture, $2-3 \mathrm{~cm}$ lateral to the midline, beneath a small $C$ shaped scalp flap. After skin closure, the Rickham reservoir was cannulated with a butterfly needle, which was then connected to a disposable closed drainage system. Two patients underwent insertion of Rickham reservoirs into the occipital horn.

Ventriculostomy catheters (Forth Medical Limited; Newbury, Berkshire, UK) were also placed into the frontal horn of the ventricles. The distal end of the $25 \mathrm{~cm}$ long catheter was tunnelled beneath the scalp and brought through a separate stab incision 3 to $4 \mathrm{~cm}$ lateral to the linear incision created for the burr hole, ${ }^{610}$ before being connected to a closed ventricular drainage system. Two patients had ventriculostomy catheters inserted into the occipital horn. 
Indications for ventricular drainage. Depicted are the diagnoses leading to ventricular drainage as the \% of total number of drains placed.

$S A H=$ subarachnoid

haemorrhage;

ICH/IVH = intracerebral (intracerebellar) or intraventricular

haemorrhage,

misc $=$ miscellaneous

(congenital or primary adult hydrocephalus, cerebellar infarction) Rickham = Rickham reservoirs; Ventric = ventriculostomies.

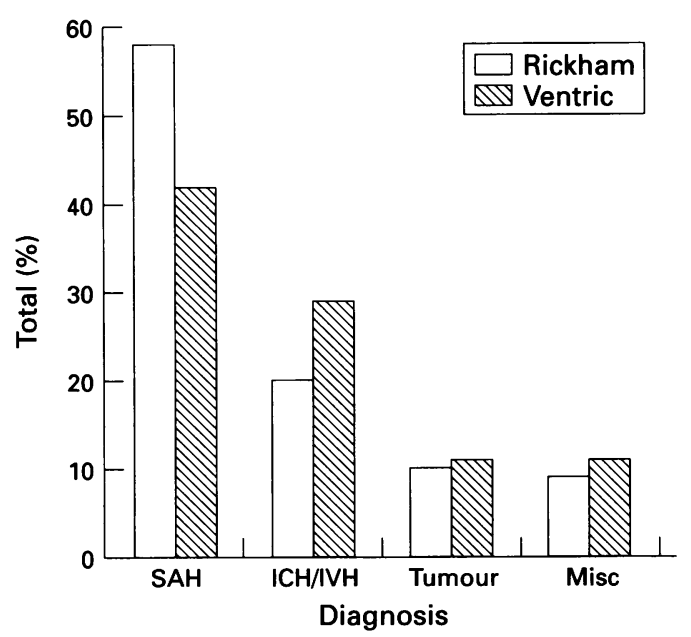

All procedures were undertaken in the operating theatre under sterile conditions. Specimens of CSF were routinely analysed on a daily basis. The technique of specimen collection was identical in both methods of ventricular drainage. As recommended by Mayhall et $a l,{ }^{6}$ only positive cultures either of CSF or of the catheters themselves were used for diagnosis of infection. The day of infection refers to date of sample collection.

The mean age of patients with Rickham reservoirs was 57 (range 2-83) years; the mean age for the ventriculostomy catheter group was 54 (range 14-80) years. Thirty seven women and 24 men had Rickham reservoirs; 32 women and 29 men had ventriculostomy catheters.

Hydrocephalus after subarachnoid haemorrhage was the most frequent reason (figure) for both Rickham reservoirs (59\% of cases) and for ventriculostomy catheters (42\%). Other indications were less common (figure).

Two sided tests of significance with $\mathrm{Z}=0.05$ were performed with the critical ratio with continuity correction method. ${ }^{11}$

\section{Results}

In this retrospective review, 18 of $67(27 \%)$ of Rickham reservoirs and seven of 70 ventriculostomy catheters $(10 \%)$ were found to be infected. This difference in the infection rates was statistically significant $(p<0.015)$; the $95 \%$ confidence limits on the difference were $4.5 \%$ to $30 \%$.

Possible risk factors associated with infection were examined. If the variables age and diagnosis were included in a logistic regression analysis along with the type of drain, only Rickham reservoirs were found to be associated with an increased risk of infection $(P<$ $0.015)$. Furthermore, the average duration of ventricular drainage before identification of positive cultures was similar for both methods: 5.7 days for Rickham reservoirs and 6.0 days for ventriculostomy catheters. Of the infected Rickham reservoirs, nine occurred in women and nine in men. Infection of ventriculostomy catheters occurred in two women and five men. Antibiotics were given during CSF drainage with $79 \%$ of Rickham reservoirs and $54 \%$ of ventriculostomy catheters, but all patients harbouring infected Rickham reservoirs and six of the seven patients with infected ventriculostomy catheters were being treated with antibiotics at the time of positive cultures. The type of antibiotic did not differ significantly between the two groups: cephalosporins and penicillins were used.

Other potential risk factors examined included concurrent cranial operations and mortality. Five of $18(28 \%)$ infections of Rickham reservoirs were associated with craniotomies - that is, the craniotomies were performed within 10 days before reservoir placement or during the time of ventricular drainage, but 12 patients operated on under the same circumstances were not infected. One infection of a ventriculostomy catheter occurred in a patient who underwent a LeFort I procedure for a clival chondrosarcoma and was managed postoperatively with prolonged lumbar drainage. Sixteen patients with uninfected ventriculostomy catheters underwent cranial procedures. There were 10 patients treated with Rickham reservoirs who died during the same stay in hospital; of these patients, three developed reservoir infection $(30 \%)$, as opposed to 20 patients with ventriculostomy catheters dying of whom two developed infection (10\%). None of the deaths were directly attributable to infected drains. Thus none of the factors examined other than type of catheter seemed to be related to an increased infection rate, and, moreover, none of the factors examined could account for the increased rate of infection of Rickham reservoirs.

After identification of positive cultures, 14 Rickham reservoirs were removed at a further operation. Four infections of Rickham reservoirs were managed with antibiotics alone (intravenous with or without intrathecal). Of the second, one reservoir was used in the conversion to a ventriculoperitoneal shunt. Infections of ventriculostomy catheters were managed by immediate removal and antibiotics in two cases; and with antibiotics without immediate removal of the catheter in five cases.

Most infections both of Rickham reservoirs and of ventriculostomy catheters were caused by a coagulase negative staphylococcus, $54 \%$ and $63 \%$ of microorganisms respectively (table 1). Six Rickham reservoirs were infected with two microorganisms; multiple organisms were not encountered in the ventriculostomy catheter group (table 2).

\section{Table 1 Distribution of infective microorganisms}

\begin{tabular}{lrl}
\hline & Rickham & Ventriculostomy \\
\hline Coagulase negative staphylococcus & 14 & 5 \\
Staphylococcus aureus & 4 & 1 \\
Streptococcus species & 4 & 0 \\
Diphtheroids & 1 & 0 \\
Acinetobacter & 1 & 0 \\
Coliforms & 1 & 0 \\
Enterobacter & 0 & 1 \\
\hline
\end{tabular}


Table 2 Pattern of infection of ventricular drainage catheters

\begin{tabular}{lllll}
\hline & $\begin{array}{l}\text { Coag staph } \\
\text { alone }\end{array}$ & $\begin{array}{l}\text { S aureus } \\
\text { alonet }\end{array}$ & Other alone $\ddagger$ & $\begin{array}{l}\text { Coag staph }+ \\
\text { others }\end{array}$ \\
\hline Rickham & 7 & 3 & 2 & 7 \\
Ventriculostomy & 5 & 1 & 1 & 0 \\
\hline
\end{tabular}

*Number of drains infected with coagulase negative staphylococcus alone.

tNumber of drains infected with staphylococcus aureus alone.
\pm Number of drains infected with a single species of microorganisms indicated in table 1 .

\$Number of drains infected with a single species of microorganisms indicated in table 1 . ism among those indicated in table 1 .

\section{Discussion}

In this comparison of the infection rates in two methods of ventricular drainage, a positive microbiological result was used as the criterion for infection, as recommended by Mayhall et al. ${ }^{6}$ With this criterion, Rickham reservoirs were found to be associated with a significantly higher rate of infection than ventriculostomy catheters. The rates of infection of the two methods, $27 \%$ and $10 \%$, respectively, fall within previously reported infection rates for ventricular drains. ${ }^{1} 46-9$

None of the other variables examinednamely, age, sex, diagnosis, duration of ventricular drainage, concurrent craniotomy, or mortality were correlated with an increased risk of drain infection. Furthermore, there was no additional evidence from case note review-for example, immunosuppressionthat those undergoing placement of Rickham reservoirs were more prone to infections than those with ventriculostomy catheters.

The distribution of offending microorganisms in this series, which is skewed towards Gram positive cocci, is similar to that described by Wyler and Kelly, ${ }^{9}$ but differs from the series of Mayhall et al where a significant percentage of infections were associated with Gram negative microorganisms. ${ }^{6}$ A possible basis for this difference may be related to exclusive use of nafcillin in their series. Interestingly, only Rickham reservoirs were affected by more than one organism. The present study does not provide support for the efficacy of prophylactic antibiotics.

The predominance of a coagulase negative staphylococcus suggests that contamination with skin flora is a mechanism of infection. The increased infection rate of Rickham reservoirs may be associated with the greater frequency of manipulation required in their management. Percutaneous drainage employing a butterfly needle inserted into the Rickham reservoir necessarily involves a risk of contamination with skin flora: the needle is often dislodged during drainage, and requires skin preparation and reinsertion. Ventriculostomy catheters, on the other hand, are inserted only in the operating theatre and are not manipulated elsewhere except to obtain CSF specimens. It may be that the tunnelling technique is associated with a lower infection rate. ${ }^{10}$ Finally, once the Rickham reservoir is contaminated, only 5 to $6 \mathrm{~cm}$ of tubing separates this from the ventricle. On the other hand the ventriculostomy catheter is $25 \mathrm{~cm}$ from the ventricle to the connection with the closed drainage system, and this may delay the migration of contaminating microorganisms. The similar duration from ventricular drainage to infection for both methods, however, suggests that catheter infection arises primarily from contamination by skin flora at insertion.

As well as an increased frequency of infection, a further disadvantage of Rickham reservoirs is that once infected, they must be removed in the operating theatre, although general anaesthesia is not always necessary. By contrast, ventriculostomy catheters are removed at the bedside.

Given these findings, we recommend the use of ventriculostomy catheters for routine CSF drainage, because the lower infection rate reduces morbidity, and its attendant costs. In cases where more prolonged drainage is required we share the view that ventriculostomy catheters should be changed after six days. ${ }^{\circ}$

We thank G Alexander West for his critical reading of this manuscript and B Hazell-Smith for secretarial assistance.

1 Bering EA. A simplified apparatus for constant ventricular drainage. $\mathcal{F}$ Neurosurg 1952;8:450-2.

2 Lundsberg N, Troupp $\mathrm{H}$, Lorin $\mathrm{H}$. Continuous recording of the ventricular-fluid pressure in patient with severe of the ventricular-fluid pressure in patient with severe acute traun $581-90$.

3 Kusske JA, Turner PT, Ojemann GA, Harris AB. Ventriculostomy for the treatment of acute hydrocephalus following subarachnoid hemorrhage. $\mathcal{f}$ Neurosurg 1973 38:591-5.

4 Narayan RK, Kishore PRS, Becker DP, et al. Intracranial pressure: to monitor or not to monitor. $\mathcal{f}$ Neurosurg 1982;56:650-9.

5 Rickham PP, Penn IA. The place of the ventriculostomy reservoir in the treatment of myelomeningoceles and hydrocephalus. Dev Med Child Neurol 1965;7:296-301.

6 Mayhall CG, Archer NH, Lamb VA, et al. Ventriculostomy-related infections: a prospective epidemioculostomy-related infections. a prospective

7 Ratch neous cerebrospinal fluid reservoir. $N$ Engl $\mathcal{F}$ Med 1968;

8 Smith RW, Alksne JF. Infections complicating the use of external ventriculostomy. F Neurosurg 1976;44:567-70.

9 Wyler AR, Kelly WA. Use of antibiotics with external ventriculostomies. $\mathcal{F}$ Neurosurg 1972;37:185-7.

10 Black P McL, Ojemann RG. Hydrocephalus in adults. In Youmans, JR, ed. Neurological surgery. Philadelphia: WB Saunders, 1990:1284.

11 Colton T. Statistics in medicine. Boston: Little, Brown and Co, 1974:163-9. 